\title{
Yongquan Maneuver'S Oddysey: Current Validation Of Its Significance Of P Through The Fisher's Exact Test For Dichotomous Variables
}

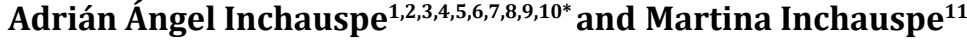 \\ ${ }^{1}$ Invited Foreigner Professor - China National Academy of Medical Sciences,China \\ ${ }^{2}$ Editorial consultant of the Medical University of Hainan,Haikou, Hainan, China \\ ${ }^{3}$ Editorial Board Member of the Journal of Acute Disease (JAD),Haikou, Hainan, China \\ ${ }^{4}$ Editorial Highlight Member of the World Journal of Critical Care Medicine,Baishideng Pub. , Beijing, China \\ ${ }^{5}$ Editorial Guest Member of Frontiers of Clinical Pharmacology Research and Outcomes Journal,Basil, Switzerland \\ ${ }^{6}$ Academic Member of the World Cardiothoracic-Renal Disease, Chongqing University, China \\ ${ }^{7}$ Scientific Department, Argentina Acupuncture Society, Buenos Aires, Argentina \\ ${ }^{8}$ Argentina Resuscitation Council, Buenos Aires, Argentina \\ ${ }^{9}$ Investigation Department, HINEA y C. "Dr. Alejandro Korn", Melchor Romero, La Plata, Argentina \\ ${ }^{10}$ Professor of the Medical Sciences Faculty of La Plata University, La Plata, Argentina \\ ${ }^{11}$ Technical Bio-Statistical Advisor \\ *Corresponding Author: Adrián Ángel Inchauspe, School of Medicine, La Plata University, Argentina.
}

Received: May 08, 2019; Published: July 26, 2019

DOI: $10.31080 /$ ASPE.2019.02.0114

\begin{abstract}
Aim: After thirty years saving clinical dead patients, both author and collaborative provides a profound methodological -statistical analysis achieved by KI-1 Yongquan resuscitations.

Methods

The KI-1 Yongquan complementary resuscitation maneuver

Yongquan action over the heart physiology: The author has already found out that after the stimulus over KI-1Yongquan -the first point in the Kidney Meridian -many cases with failure of basic and advanced resuscitation returned back to life using this ancient technique, which seemed to be a legend established along millennia in the Far East had a normal cardiac rhythm and regained consciousness [3,8].
\end{abstract}

Likewise, technical advisor Martina Inchauspe demonstrated the validation of this CPR- complementary maneuver, deepening its significance of certainty respect to current techniques and protocols still in force.

Maybe such assertion led the Chinese to conclude that Yongquan had the ability to "reset" the vital signs that are absent, as a battery that would provide us with a source of alternative vital energy if our own existence were in severe danger [6].

Yongquan's action over the brain physiology: Regarding K-1 Yongquan application as a CRP revival point, divulgation was not limited to actuarial cardiac results, but KI-1 Yongquan function as a brain protector in both traumatic and vascular brain injury situations should be included. Acupuncture is actually convoked for supporting Western Medicine to help patients with serious neurological recovery. Traditional Chinese Medicine Qi balancing effect principle can improve cognitive, intellectual and psychomotor patterns even after severe brain injury. Needless to say, all patients subjected to the stimulation of KI-1 Yongquan by cardiac arrest were neurologically classified with 3 points on the Glasgow Scale [8].

Results: Application of statistical analysis was based upon the index Paradigm [12].

The difference obtained was also confirmed to be statistically significant, adding to this analysis the F-test for dichotomous variables; thus, all the statistical validations demonstrated once more the relevant certainty before other methods currently used instead of K-1Yongquan [5].

Today, in 89 patients in impending death situations for stroke or cardiac arrest, even in extreme critical impairment (skull fracture crushed chest, etc.), KI-1 Yongquan helped vital organs and consciousness to recover, and failed to modify natural evolution in only 14 cases.

Conclusion: Randomness constitutes an optimum objectivity condition; but associated with unpredictable results. Then, the "alternative hypothesis" will found the research with an impartial criterion.

Keywords: KI-1 Yongquan; Resuscitation Maneuver; Cardiac Arrest; Stroke; CPR Failure Resource; Brain Injury’s Protection 


\section{Core tip}

The K-1 Yongquan complementary resuscitation maneuver, systematized since 1987, has been consistently performed in sudden death and cardiac arrest conditions as a final resource upon both basic and advanced CPR failure. After almost thirty years of experience, the author herein provides a reasoned survival bio-energetic circuit based on a detailed methodological -statistical analysis of the Wondrous Vessels (Qi jing ba mai) participating in it.

The divulgation of K-1 emergency therapeutic possibilities looks for its inclusion into Critical Care Protocols, in order to upgrade survival rates in both cardiac arrest and stroke victims worldwide.

\section{Background}

I was born in La Plata, capital city of our Province, Buenos Aires. Nevertheless, immediately after being born, I was taken to Berazategui, to breathe the air of my true and beloved homeland. I have lived there all my life, surrounded by the relatives of those who were neighbors and friends not only of my parents but also of my grandparents. Hence, was from there where I was kidnapped by terrorists in 1973. I was only 10 years old [1].

I was the youngest grandson of a wealthy businessman who worked in the textile industry logistics. I was deprived of my freedom with no judgment or reason whatsoever, and at such short age I was obviously unaware of the risk of dying. I lost my innocence harshly and abruptly, when I realized about the danger I was being exposed to, without the least possibility of defending myself.

Abducted from my home for 30 hours until the ransom was paid - something which I am nowadays thankful for, as it put an end to my hardships - I was forced many times to be away from my home. Three months later, my own father was also kidnapped, which definitely left our entire family broke - as we incurred into huge debts that were finally cancelled during my training as surgery resident - and which left me with a constant unpleasant feeling of deep insecurity.

Before the end of that same year, the students of a neighboring town and their relatives had to be evacuated from my school after a bomb threat. My parents had to hide me for weeks due to the siege of anti-subversion groups, who were looking for survivors from terrorist attacks, to recognize possible revolutionary extremists to illegally execute them.

In the face of such scenario, I needed to embrace the path of martial arts still very young, trying to recover my damaged confidence, which usually "derailed" after frequent panic attacks, as I suffered imaginary pursuits that increased my fear of being executed.
Luckily, I found excellent teachers; wise and really understanding people that were able to "put back on rails" my self-esteem and confidence. That meant not only training in self-defense techniques, but also thoroughly studying the principles of Oriental Medicine, which allowed me to learn about vulnerable points, many of which were also important points in Acupuncture. In the East, everything contains in itself the essential nature of its opposite: the same points that were used to knock someone unconscious were the same ones that enabled them to regain consciousness. As with the Yin and Yang, everything I learnt undoubtedly showed both sides of the same coin [1].

Once persecution and urban terrorism were finally over in Argentina, I could devote all my efforts to learning about the impact that this millenary knowledge had over health and several pathological states. My focus was then trying to verify this old Eastern knowledge with present western medical principles, and to base my findings upon scientific evidence to fully comprehend through a deep logical justification everything what I learnt.

\section{Introduction}

After reading "Zen in the Martial Arts" by Taisen Deshimaru [2], I learnt about a specific point on the sole of the foot - in Japanese, Koson -, which had the ability to bring back both consciousness and vital signs of a person in a critical condition. The description did not explain why the point had such effect. Its use was presented as a very old empirical practice with presumably amazing outcomes.

It was during the spring of 1987 that the first event took place. I had started my General Surgery residence in June that same year and during my days on-call I was part of a CPR rescue team at Dr Rodolfo Rossi Hospital.

Some time after 10:30 p.m. we received a call from the hospital ER. When we arrived at the shock-room, we were informed by the cardiologists on call, Adriana Tricerri and Guillermo Mulinaris, that the patient was with electromechanical dissociation - a condition nowadays known as pulseless electrical activity - and that he was not responding to precordial thumps.

At that time it was common practice to request the presence of a surgeon to start an IV line to measure the central venous pressure, replace fluids and deliver vasoactive substances that may reverse the patient's condition. Then I changed turns with my colleagues, compressing and insufflating the patient, in accordance with the life support protocols "action chain".

Some minutes later, the cardiologists decided to defibrillate the patient, hoping to replace the ineffective cardiac automatism with a useful rhythm that may help the heart pump blood properly. After several attempts - and almost 28 minutes trying to rescue the patient - they both decided that there was nothing left to do. Dr 
Tricerri went out to break the bad news to the patient's relatives and Dr Mulinaris started to remove the electrocardiograph off the patient. I then asked him if I could please try to recover the patient with a maneuver based on the principles of Chinese Medicine...

Method: The KI-1 Yongquan complementary resuscitation maneuver

\section{Yongquan action over the heart physiology}

I believed such outcomes could not lack proper foundations in our own knowledge, so I decided to thoroughly study this point in order to unravel the mysteries of its incredible ability to restore vital signs that have disappeared, a practice which is commonly inappropriately known as "resuscitation". Nothing to do with any religious practice, it consist in a set of actions aimed at backing up what we nowadays call "life support", that is, a series of actions that may help patients maintain their vital signs even in the case of cardiac arrest [3-5].

By the time I was starting to determine the wonderful effects of this point, I found myself in that shock-room at Dr Rodolfo Rossi Hospital, asking the professional in charge if I could try the maneuver I had created over that resuscitation point the Chinese called Yongquan or Spring Water [3].

I had already found out that Yongquan was the first point in the Kidney Meridian. The Chinese said that the Kidneys were the "prenatal Emperor", and in fact, it is the most active organ during the gestation period [4]. Moreover, the pronephros shares, during embryological development, the urinary tract with the allantois, which will carry gametes for the reproductive function, something which is taken into account by the Chinese, who have given the kidney the power to store Yuan, the essential or original energy of a human being. In other words, the ancient Chinese understood that the Kidney and the gonads had the human chromosomal potential needed for the perpetuation of our species [4]. I then inferred that we had the specific energy we needed to restart a new phase of resuscitation...

Maybe such assertion led the Chinese to conclude that Yongquan had the ability to "reset" the vital signs that are absent, as a battery that would provide us with a source of alternative vital energy if our own existence were in grave danger [6]. In fact, all my publications refer to this discovery, which can also be justified through a mathematical study that explains the electro physical bases of lightning [7].

So that spring night of the year 1987, Dr Guillermo Mulinaris with a skeptical, gentle and surprised face - told me in a low voice:

'Well, the patient has just been declared dead...if you want, you can try'.
Seized with a great emotion, not only because I was going to "resuscitate" a patient, but also because I was using such an ancient technique, which seemed to be a legend established along millennia in the Far East...

I pressed firmly the sole of the patient's foot several times and I could see, as the monitor was still connected, how electrical signals started to appear on the ECG, which indicated there was some heart activity.

- 'Let's see: stop doing the maneuver', ordered Mulinaris. And the tracing would disappear...

- 'Now, start doing it again!' said Guillermo. And I tried again, with the same outcome, but this time the ECG signals were more active and frequent.

- 'Adrián: don't stop now!' the cardiologist insisted enthusiastically, not realizing that his colleague had gone out to tell the patient's family that he was dead. My thumbs ached, so I took my fountain pen - not removing its cap so as not to hurt the patient's skin - and I pressed firmly over the point again. The electrical activity had already become stable and the rhythm seemed to be incredibly uniform and regular. I kept on doing the maneuver with formidable enthusiasm... an enthusiasm that has been intact for over 30 years.

- Just as Adriana was apologetically announcing our defeat, the patient's scream was heard from the shock-room. Adriana and the patient's relatives looked at each other desperately as one of them cried: 'It's him!!! It's him!!!'

- With a strong stimulus over Yongquan, this first patient - after 28 minutes of continuous vital support and after both basic and advanced CPR failure - had a normal cardiac rhythm and regained consciousness. He sat on the hospital bed, extubated himself and screamed...he was back to life $[3,8]$.

\section{Yongquan's action over the brain physiology}

Regarding K-1 Yongquan application as a CRP revival point, I was invited as national delegate in the $9^{\text {th }}$ International Brain Injury Association Congress in Edinburgh on $23^{\text {rd }}-26^{\text {th }}$ March, 2012 [6]. In such meeting, divulgation was not limited to actual cardiac results, but KI-1 Yongquan function as a brain protector in both traumatic and vascular brain injury situations should be included. Acupuncture is actually convoked for supporting Western Medicine, as it is generally accepted that its "Golden Points" can undoubtedly help patients during their neurological recovery. Traditional Chinese Medicine Qi balancing effect principle can improve cognitive, intellectual and psycho-motor patterns even after severe brain injury. Needless to say, all patients subjected to the stimulation of KI-1 Yongquan by cardiac arrest were in severe neurological impairment, and classified with 3 points on the Glasgow Scale [8]. 
WHO has produced statistics referred both to cardiovascular and cerebrovascular causes of death. As we can see in Table 1, the death rate due to cardiovascular causes is $23 \%$, while cerebrovascular causes add $7 \%$ to the already mentioned death rate. They all account for more than $30 \%$ of all global causes of death $[9,10]$.

\begin{tabular}{|c|}
\hline Overall causes of death \\
\hline $1^{\text {st }}$ cause: Cardiovascular diseases -------- 23\% \\
\hline $2^{\text {nd }}$ cause: Vascular brain injury-------- 7\% \\
\hline Joint percentage -- \\
\hline
\end{tabular}

Table 1: WHO statistics positioned cardiac arrest and stroke as the two most frequent of overall causes of death $[6,10]$.

\section{Methodological approach}

Chinese Medicine also calls it the "root point" of its deepest energetic plane, the Shao Yin, which comprises the Heart and both Kidneys. It is an axis that is composed of two opposing polarities - Yin and Yang; Water and Fire - needed in the combined and general interaction of the Five Elements of Chinese Medicine [4].

In the year 2010 I already found it strange that the Theory of the Five Elements - which governs Oriental (Chinese, Japanese, Korean and Hindu) Medicine along with Yin/Yang - should "casually" be homonyms to those described by Euclid in his posthumous work The Elements. In such work, this genius mathematician stated that it was only possible to conceive the Five Regular Polyhedra within nature, proving their existence in an exact manner by means of relatively simple calculations. Thus, I believed it is probable that the efficacy of these "alternative therapies" (both in terms of diagnosis and therapy) should be supported by eternal laws that do not depend on mere trial and error knowledge, but that are based on the oldest formal science: Geometry (see Figure 1,2) [11].

Comparison between Euclid's Five Regular Polyhedra (Figure 1) and the Five Elements or Movements in TCM (Figure 2) [11].

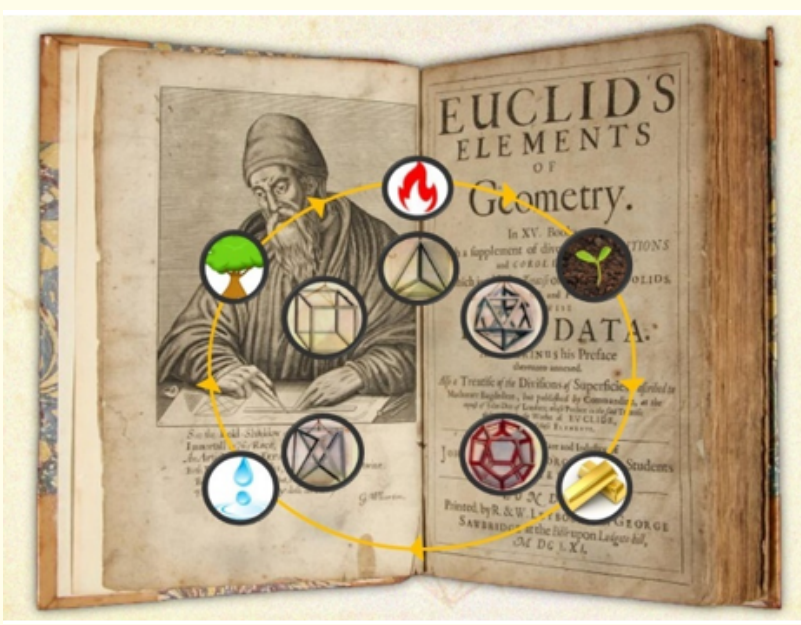

Figure 1: Euclid Five Regular Polyhedra.

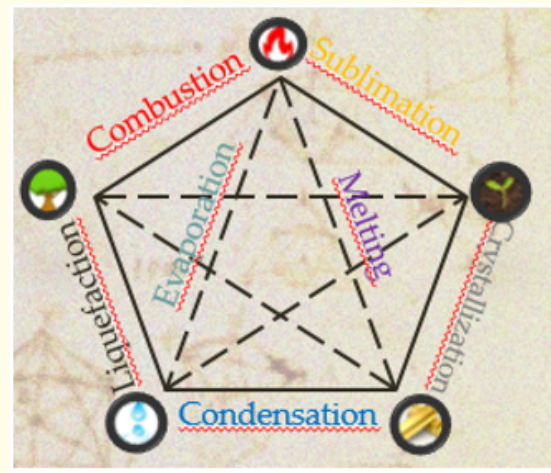

Figure 2: Five Movements in Chinese Medicine.

That proposal was presented at the $2^{\text {nd }}$ International Congress on Traditional and Alternative Medicine in Beijing and at the Chinese National Academy of Medical Sciences, before Deputy-Director, Prof. Huang Hui. At said Congress, an amusing comment was made by Dr Ning -Sun Yang, dean of Sinica Accademia in Taiwan:

'The truth is, we don't know if we have proof to say that it is like this. But, on the other hand...why can't we say that it is like this???'

Our patient was admitted to the Intensive Care Unit and for two weeks all the residents would jokingly draw a dot on the sole of the foot...just to know where to press in case of emergency.

Years have gone by and case study - 14 deaths out of 89 cases - has proven that extra-hospital survival rates are 8 times higher than the best out-of-hospital survival rates.

The model used to verify its statistical validity - prior consultation with Professor Carlo Guinzburg from the University of Bologna - was the Retrospective Cohort Study, which redeems the semiotic paradigm: the same one that gave rise to medical semiotics. According to Professor Guinzburg, such epistemological model has its roots in the times when men lived off hunting, tracking their prey and predicting its features based on its footprints (see drawing in Figure $3[9,10]$. Also called for that reason "semiotic paradigm" constitutes the foundation of the classical semiologic examination of the Medical Clinic.

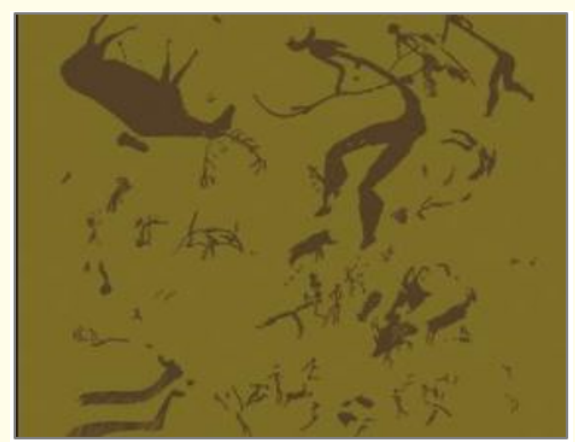

Figure 3: Prehistoric hunters. Courtesy of Book: Prehistoria. 1 largo camino de la humanidad. Author: V.M.Fernandez Martinez [10]. 
As to its statistical verification, several sequences of survival rates were presented, the first 7 of which were published in Health (2015) [9], the $8^{\text {th }}$ one in the World Journal of Critical Care Medicine (2016) [12] and the $9^{\text {th }}$ and last sampling, at the Health Care Summit Congress in Dublin (June 2018 - see Figure 4) [10]. Its value actually strives in the differential detail if the deceased patients group is considered the control group instead of the patients that may be deceased group. Thus, the possibility of combining the indiciary or semiotic paradigm with the Retrospective Cohort Study allows us to manage potential lethal effects which are collateral to the random process in cases of extreme emergencies [10].

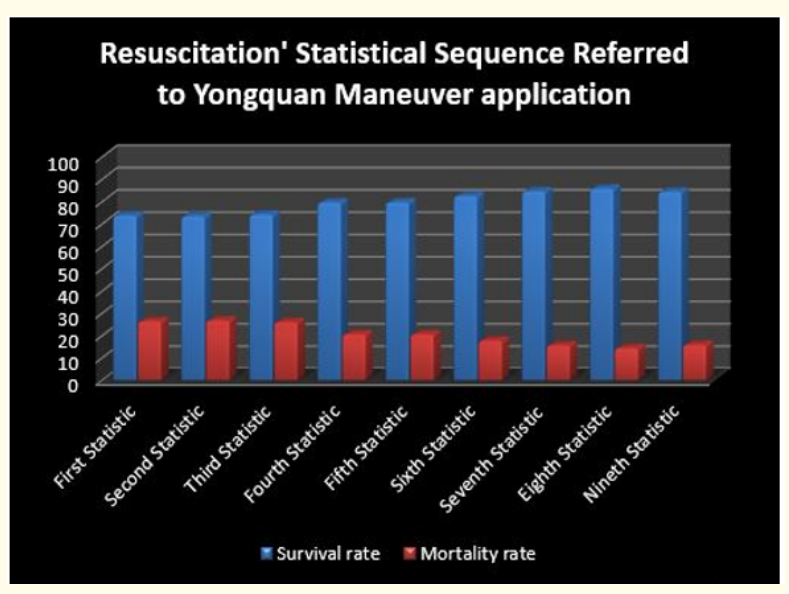

Figure 4: Resuscitation Statistical Sequence Referred to Yongquan maneuver application $[5,9,10,12]$.

This paradigm places special emphasis on individuality and originality, on qualitative features and singularity. Its validity is based upon the possibility of making deductions from the particular to the general. As I have already said, it is especially useful on those situations when there is no time available to increase the size of the sample and to move away from uncertainty.

An example of this paradigm took place during the H1N1global viral infection in 2009, when WHO declared as extreme emergency pandemia - level 6 - after 49 deaths in Veracruz, Mexico, when a risk management plan was devised, to be applied worldwide on more than 7,000 million people. Thanks to it, the plan was highly successful: the number of victims fell from 72,000 in 2010, to 607 in 2014 [10].

\section{Results}

Application of statistical analysis based on the index Paradigm [12]

As was the case while analyzing the Yong quan maneuver statistical tendencies-in spite of what has been so far stated, we have assigned priority to what is singular and of qualitative nature. As such, the proposal of a Retrospective Cohort Study model has been able to prove the following suggested affirmation as published in WJCCM May 2016 ISSN 2220-3141 (online) [12].
In statistics, a null hypothesis (Ho) is one made up with the purpose of supporting another, alternative one (Ha). Any medical study necessarily implies determining an association between such two variables under research. Any statistical treatment contemplates the possibility of a standard error (SE). That is why, the wider the sample, the more precise it becomes; consequently the possibility of results being random is greatly reduced [this analysis was previously published in WJCCM August 2013 - ISSN 2220-3141 (online) [5].

So technical advisor Ms. Martina Inchauspe demonstrated the validation of this CPR- complementary maneuver with additional statistical specific tests, deepening its significance of certainty respect to current techniques and protocols still in force.

We have 89 patients in total in which the Yonquan maneuver was tested, of which 75 survived and 14 died. In order to compare this data with the percentages of survivors in the other maneuvers, we stipulate the assumption that if 89 patients are the $100 \%$ of the sample, and how many patients would survive if the survival rate is $6.4 \%$ in CPR (Table 2; Figure 5), 30\% in defibrillation (Table 3; Figure 6) and $48 \%$ in CPR + defibrillation (Table 4; Figure 7). In this way we obtained the approximate values of patients that would survive when applying these resuscitation maneuvers, which Martina represented in round figures to be able to do the test. To the total of patients ( 89 cases) we subtracted the survivors and obtained those that would not survive.

\begin{tabular}{|c|c|c|c|}
\hline & $\begin{array}{c}\text { Out of Hospital } \\
\text { Cardiac Arrest }\end{array}$ & Yongquan & $\begin{array}{c}\text { Marginal } \\
\text { Row Totals }\end{array}$ \\
\hline Survivors & 6 & 75 & 81 \\
\hline Non-Survivors & 83 & 14 & 97 \\
\hline $\begin{array}{c}\text { Marginal } \\
\text { Column Totals }\end{array}$ & 89 & 89 & $\begin{array}{c}178 \text { (Grand } \\
\text { Total) }\end{array}$ \\
\hline
\end{tabular}

Table 2: Sample A: Out of Hospital Cardiac Arrest vs Yongquan.

The Fisher exact test statistic value is $p<0.0001$. The result is extremely statistically significant

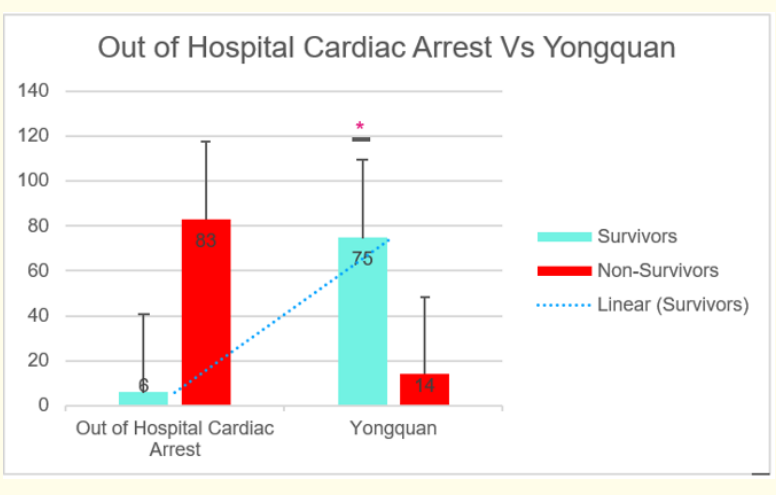

Figure 5: Out of Hospital Cardiac Arrest vs Yongquan Survivors' tendency. 
Thus we then obtained the format of the tables to perform the exact Fisher test. Martina did this test with the help of a statistical processor; and their results are on the side of each table.

The obtained result in a valuation of $p<0.0001$, considered "extremely statistically significant". Martina did it with the Graph Pad site, with a two-tailed P value, which they recommend to analyze these cases with dichotomous variables for being more reliable.

\begin{tabular}{|c|c|c|c|}
\hline & Defibrillation & Yongquan & $\begin{array}{c}\text { Marginal } \\
\text { Row Totals }\end{array}$ \\
\hline Survivors & 27 & 75 & 102 \\
\hline Non-survivors & 62 & 14 & 76 \\
\hline $\begin{array}{c}\text { Marginal Column } \\
\text { Totals }\end{array}$ & 89 & 89 & $\begin{array}{c}178 \\
\text { (Grand } \\
\text { Total) }\end{array}$ \\
\hline
\end{tabular}

Table 3: Defibrillation vs Yongquan.

The Fisher exact test statistic value is $p<0.0001$. The result is extremely statistically significant

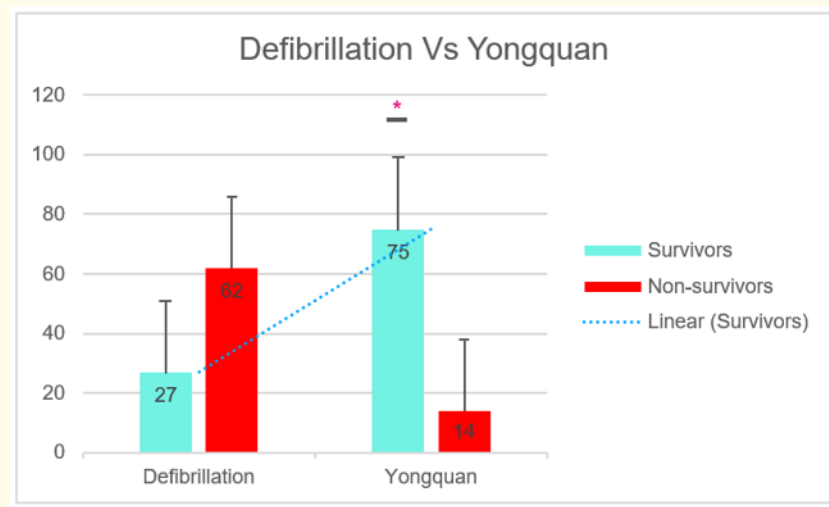

Figure 6: Defibrillation vs Yongquan Survivors' tendency.

Martina remarked that the difference here is also confirmed to be statistically significant; thus, all of the considerations from the previous example are valid, demonstrating once more the comparative value of the Yong quan method [5]

\begin{tabular}{|l|c|c|c|}
\hline & $\begin{array}{c}\text { CPR + } \\
\text { Desfibrilación }\end{array}$ & Yongquan & $\begin{array}{c}\text { Marginal } \\
\text { Row Totals }\end{array}$ \\
\hline Survivors & 43 & 75 & 118 \\
\hline Non- Survivors & 46 & 14 & 60 \\
\hline $\begin{array}{l}\text { Marginal } \\
\text { Column Totals }\end{array}$ & 89 & 89 & 178 \\
(Grand Total)
\end{tabular}

Table 4: Sample B: CPR + Defibrillation vs Yongquan.

The Fisher exact test statistic value is $p<0.0001$. The result is extremely statistically significant.

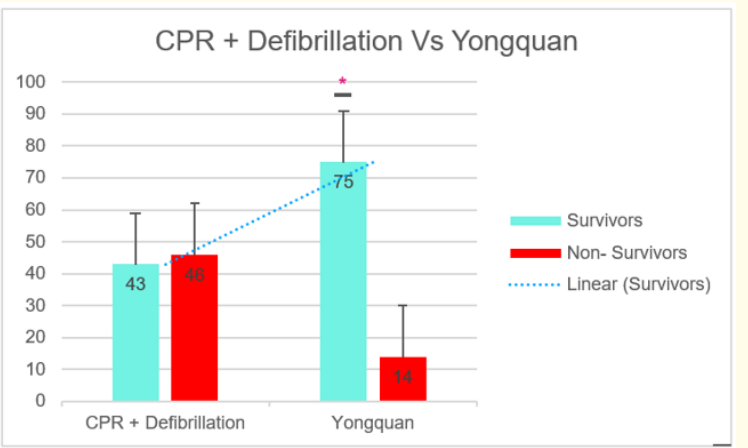

Figure 7: CPR + Defibrillation vs Yongquan Survivors' tendency.

Compared to with reported survival rates - less than $6,4 \%$ in extra hospital rescues and 19\% in the best Emergency Units - K-1 application results are quite over this average (89 survivors and 14 deceased $=84.27 \%$ survival rate). Of them, 52 cases suffered a stroke after severe hyper or hypotension episodes - among other kinds of brain injuries (trauma) - meaning only $15.73 \%$ of overall deaths are left out of this trial [10].

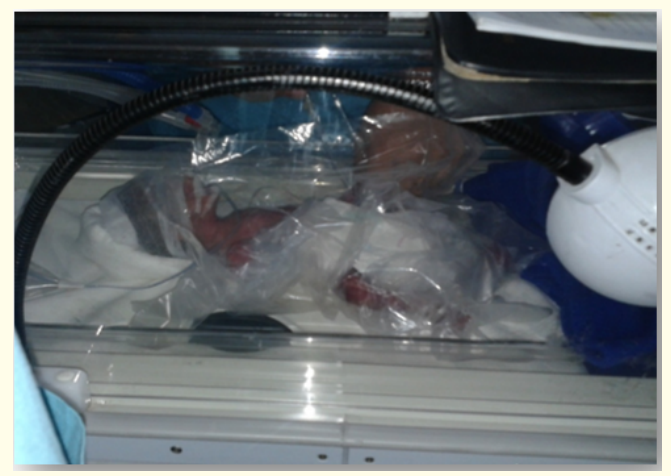

Figure 8: 700 grams premature newborn baby with acute Staphylococcus meningitis rescued by K-1 Yongquan maneuver [10].

\section{Discussions}

Approximately 135,000,000 deaths from cardiovascular causes (AHA) are registered every year worldwide, as well as a proven increase in the overall prevalence of coronary heart disease. Currently, the incidence of out-of-hospital cardiac arrest ranges from 20 - 140 cases per 100,000 people, and their survival ranges from 2 to $12 \%$ [11]. In USA, more than 500,000 children and adults suffer cardiac arrest, of which only recover - despite its extreme preventive measures - a figure of less than 15\% [12].

As was the case while analyzing the Yong quan maneuver statistical tendencies-in spite of what has been so far stated, we have assigned priority to what is singular and of qualitative nature. As such, the proposal of a Retrospective Cohort Study model has been able to prove the following suggested affirmation as published in WJCCM May 2016 ISSN 2220-3141 (online) [5]. By this way, the application of statistical analysis was based upon the semiotic para$\operatorname{digm}[9,10]$. 
Regarding jointly taking cardiovascular and cerebrovascular mortality rates, they find in hand, suffering a stroke of Oriental Medicine a plausible explanation that can be totally extrapolated to Western Medicine. Remember that when suffering from a cardiac arrest, it is very possible that cerebral hypofluxs hould generate a cerebrovascular accident. On the other hemorrhagic etiology can also be the origin of a cardiac arrest. As we can see, one is the cause and/or the consequence of each other, thus this relationship can be located since millennia within the Tao diagram $[6,10,13]$.

There is proven experimental scientific evidence of the therapeutic possibilities of Acupuncture in brain-damage. Babinski plantar cutaneous reflex and its utmost semiological value prove it. After age 2, hallux extension results patognomonic of a cortexpyramidal lesion, which can be tested only with a pin scratch on the sole of the foot (see Figure 5,6). This reliable indicator can be matched with the K-1 Yongquan Resuscitation Maneuver which, with a simple pressure between the anterior and middle third of the sole, allows vital signs to recover with a proved effectiveness with neurological protection $[6,10]$.
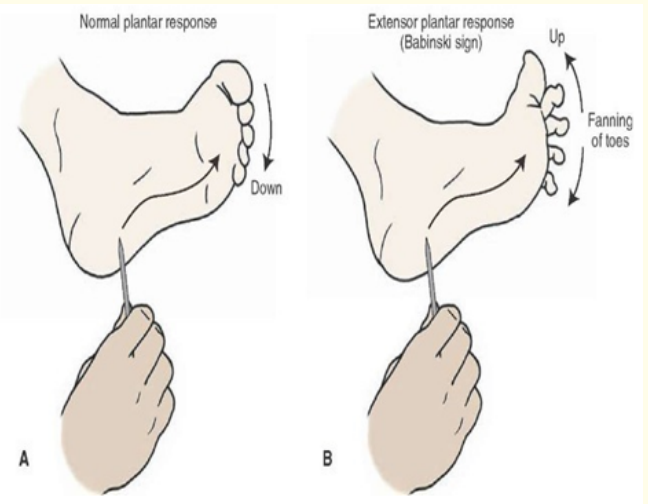

Figure 9: Illustration of Babinski plantar cutaneous reflex. Source: http://what-when-how.com/neuroscience/the-uppermotor-neurons-motor-systems-part-3/ [6].

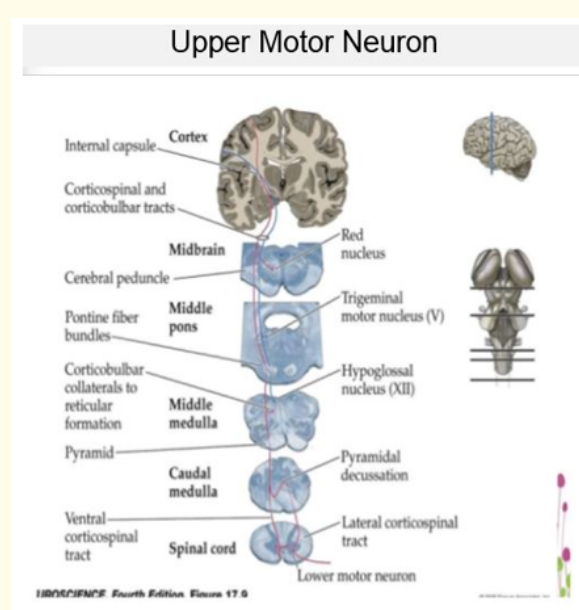

Figure 10: Upper Motor Neurone Syndrome Source: Neuroscience, Fourth Ed., Fig 17.9 @2008 Sinauer Associates, Inc [6].
Taking into consideration the moral, bioethical and legal aspects of its application, this paper represents an exceptional case, for the randomness principle-which provides the foundation to current global scientific methodology -would severely damage the control group under study.

Random non-intervention practiced on such a group would inevitably lead to a most serious ethical problem: NOT PROVIDING THE NECESSARY AND TIMELY ASSISTANCE THAT MAY SAVE A LIFE to those patients who have been "sorted out" (i.e. destined not to be benefitted by the application of the maneuver) $[5,14]$.

Article 32 of the Declaration of Helsinki VI on Ethical Principles for Medical Research Involving Human Subjects and Human Rights should not be forgotten when it states:

32. "In the treatment of a patient, where proven prophylactic, diagnostic and therapeutic methods do not exist or have been ineffective, the physician, with informed consent from the patient, physicians must be free to use unproven or new prophylactic, diagnostic and therapeutic measures, if in the physician's judgement it offers hope of saving life, reestablishing health or alleviating suffering" [15].

\section{Conclusions}

According to Sandro Palazzo in his book: "Heráclito y Parménides - Lo Uno y lo Múltiple"[16], the principle that inhabits all things from its deepest reality is understood in Greece as "truth". In this language it is called "aletheia", a word composed of the prefix of negation "a-" and its root "lath" (latent: "to be hidden"). The Greeks wanted to mean that the "truth" is what is known "exposed"; and the numbers previously quoted in this work bare the catastrophic reality. Somehow, the statistics teach us that, in the best of cases, of every 10 cardiac arrests, only one will have a chance to survive.

In the casuistry derived from our research, resorting to metachronic groups of cardiac arrest victims, the randomness unfavorability was solved reaching in that sense a P significance, totally valid as product of a correct statistical analysis $[6,10,17]$.

It will be useful to keep in mind that randomness can contribute with an optimum objectivity condition; but it can be also associated with unpredictable results. Then, the "alternative hypothesis" - as shown in this investigation - will found its deductions with an impartial criterion.

\section{Acknowledgement}

I would like to especially thank to Miss Mariángeles Pucci, ISO 9001:2015 Auditor Lead, for the revision of the format and her suggestions regarding details of the work; and to Mrs. Clara Spadafora, English Professional Translator specialized in Medical Sciences, for her invaluable and unconditional support for the successful publication of my papers. 


\section{Summary}

The K-1 Yongquan complementary resuscitation maneuver, systematized since 1987, has been consistently performed in sudden death and cardiac arrest conditions as a final resource upon both basic and advanced CPR failure. After almost thirty years of experience, the author herein provides a reasoned survival bio-energetic circuit based on a detailed methodological -statistical analysis of the Wondrous Vessels (Qi jingbamai) participating in it.

The divulgation of K-1 emergency therapeutic possibilities looks for its inclusion into Critical Care Protocols, in order to upgrade survival rates in both cardiac arrest and stroke victims worldwide.

\section{Conflict of Interest}

The author reports no conflict of interest.

\section{Bibliography}

1. Inchauspe AA. "Systematized Integration of Therapeutic Acupunctural Knowledge”. Chap. 8 "Kidney (Shen)”. Nova Publishers, Inc., New York, USA (2014): 469-474.

2. Deshimaru T. "Zen y ArtesMarciales". Luis Cárcamo Editor. San Raimundo,58. Madrid, España (1977): 104-105 - ISBN: 8485316-41-X

3. Inchauspe AA. "Traditional Chinese medicine K-1 Yongquan and Resuscitation: Another Kind of Lazarus Phenomenon". Resuscitation 81 (2010): 505-506.

4. Inchauspe AA. "Traditional Chinese Medical Criteria about the Use of Yongquan as a Life-Support Maneuver". In: Kuang, H.X., Ed., Recent Advances in Theories and Practice of Chinese Medicine, InTech Publ. Croatia (2011): 361.

5. Inchauspe AA. "Drawing the Yongquan Protocol into the Different Stages of the Cardiopulmonary Resuscitation Sequence". World Journal of Critical Care Medicine 2 (2013): 17-20.

6. Inchauspe AA. "Traditional Chinese and Brain Protection: Can Its Principles Be Consistent with Western Science?” Poster Presentation at the 9th IBIA International Brain Injury Association Congress, Edinburgh (2012).

7. Inchauspe AA. "Between Heaven and Earth" Scientific Basis of the Action of the Shao Yin: Lightning's Physical- Mathematical Analysis". Pharmacology and Pharmacy 5 (2014): 175-180.
8. Inchauspe AA. "My Experience in Cardiovascular Resuscitation through K-Yongquan". VII Congreso deFILASMA-XVII Curso Internacional de Acupuntura, Farmacopea y Medicina Tradicional China, IV Simposium SAME, Seville (2010).

9. Inchauspe AÁ. "Resuscitation on the K-1 Yongquan: Ethical and Methodological Aspects of Its Pilot Study". Health 7 (2015): 809-818.

10. Inchauspe AA. "K-1 Yongquan: Methodological and Ethical Aspects of its Pilot Study II" as keynote speaker at the 13th World Congress on Healthcare and Technologies. Dublin, Ireland (2018).

11. Romero H. "Tasa de supervivencia de RCP es más baja de lo que se piensa" La Cruz Roja de Mexico practica RCP. Mexico Mayo (2017).

12. Meaney., et al. "Calidad de la reanimación cardiopulmonar: mejora de los resultados de la reanimación cardíaca intra y extrahospitalaria Declaración de consenso de la American Heart Association". (con el apoyo del American College of Emergency Physicians.

13. Inchauspe AA. "Is Traditional Chinese Medicine Definitely an Exact Science?”. EC Dental Science 11.6 (2017): 255-277.

14. Inchauspe AÁ. "PC-9 Zhongchong as a resuscitation aid for bilateral double-amputee patients". World Journal of Critical Care Medicine 5 (2016): 143-149.

15. Article 32 of the Declaration of Helsinki VI on "Ethical Principles for Medical Research Involving Human Subjects and Human Rights".

16. Palazzo s. "Heraclito y Parménides: lo Uno y lo Multiple"; "Heráclito: vida y personalidad”; Ed. Bonalletra Alcompás, S.L. Barcelona, España (2015): 42-43.

17. Inchauspe AA. "Torsadogenic index for prevention of acute death". Journal of Acute Disease 4 (2015): 266-273.

\section{Volume 2 Issue 8 August 2019 \\ (C) All rights are reserved by Adrián Ángel Inchauspe and Martina Inchauspe.}

